\title{
Segments Assembly Process Analysis and Quality Control Measures Yuncai XIA
}

\author{
Department of Mechanical Engineering, Dalian Vocational Technical College, Dalian, Liaoning, \\ 116037, China
}

laoliu2345@163.com

Keywords: Segment, Billet, Assembly Process Control Measures

\begin{abstract}
In continuous casting, continuous casting lines constitute a single continuous casting machine is generally referred segment, In general, it is divided into actuate segment (or curved section), straightening section, horizontal section and so on. According to different billet can be divided into plate segments, billet segments and round billet segments. The most common is the production of plate and billet segments, segments that require high assembly quality, both inside and outside the arc to adjust the degree of opening height the frame and the roller arc height high demand, In this paper, analysis billet segments assembly processes and discussion of its assembly quality control measures.
\end{abstract}

\section{Introduction}

Segments having a mass production situation, the device structure is complex, the opening degree of precision and quality point arc, big impact on the quality of the slab. Size on the opening height the $A\left(A_{0}\right)$ has two aspects, one curved arc size $A_{1}, A_{2}$, and second, to adjust the opening size of $A_{3}$. For the assembly precision, high efficiency, the choice of method of assembling interchangeable assembly method. To this end find out the impact of the relevant dimensions of assembly precision, the establishment of assembly dimension chain, determine the composition of limit deviations ring. Finally, the various components of the assembly dimension chain ring with a deviation of size parts processing chain analysis and control.

\section{Effect of the Opening Height the $A\left(A_{0}\right)$ Size are the $A_{1}, A_{2}, A_{3}$, Analysis and Calculation of Tolerances and Limit Deviations of these Dimension.}

The opening height precision rolled plate according to the technical requirements for the $A=230 \pm 0.3 \mathrm{~mm}$ high precision, as shown in Figure1, Dimensions $A_{1}, A_{2}, A_{3}$ for the composition ring, choose completely interchangeable assembly method. In both ends of the roll Case No.1, 7, $A_{1}=759.48 \mathrm{~mm}, \quad A_{2}=765.41 \mathrm{~mm}, A_{3}=1753 \mathrm{~mm}$. Figure. 2 shows the assembly dimension chain. Where $A_{0}$ is for the closed loop, $A_{1}$ and $A_{2}$ are for minus loop, $A_{3}$ is for plus loop.
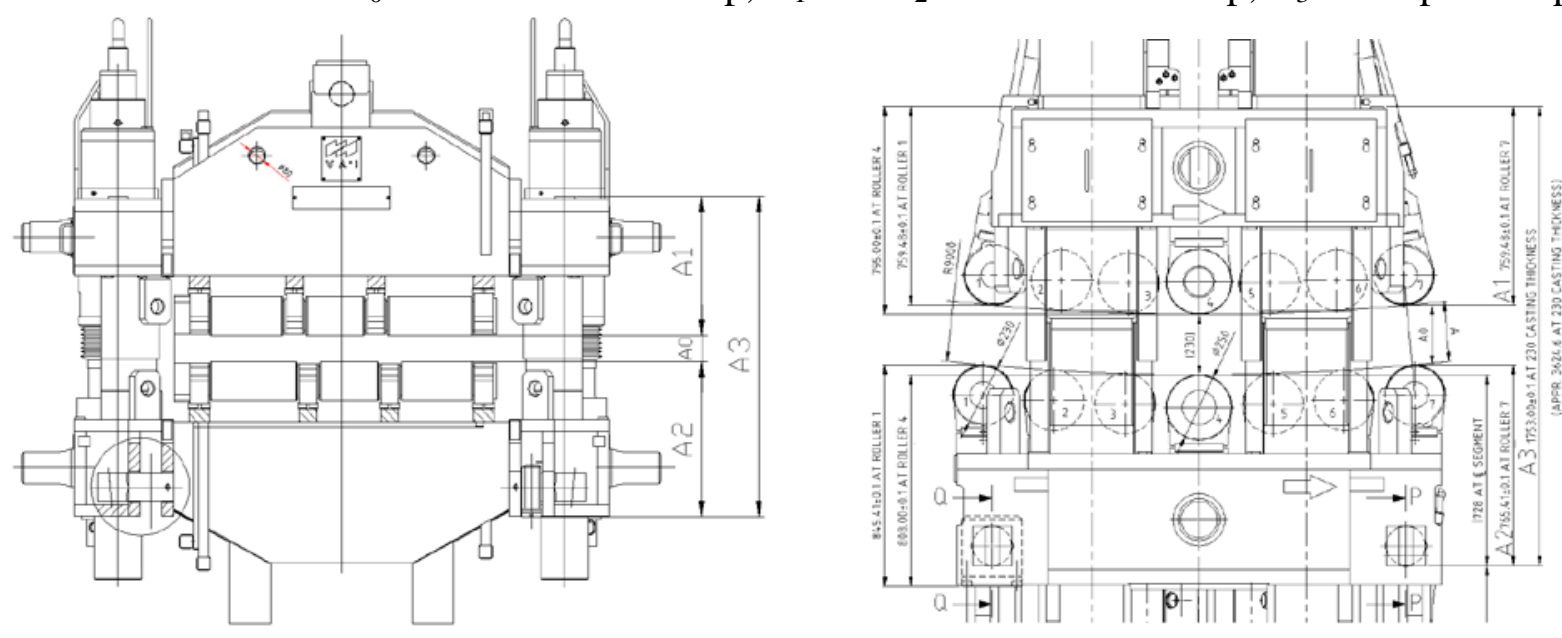

Fig.1 Segment General Assembly Drawing 


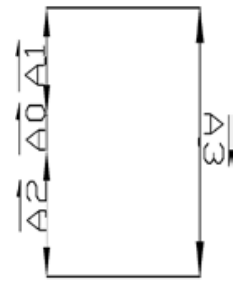

Fig.2 Segments opening height assembly dimension chain

Closed Loop Size. The closed loop size is

$$
A_{0}=A_{3}\left(A_{1}+A_{2}\right)=1753-(759.48+765.41)=228.11 \mathrm{~mm}
$$

Closed Loop Tolerance. The closed loop tolerance is

$$
T_{0}=+0.3-(-0.3)=0.6 \mathrm{~mm} \text {. }
$$

Each Consisting of a Ring Tolerance (tolerance average and standard tolerances of the constituent rings is given). The average composition of each ring tolerance is

$$
\left.T_{\text {avA }}=T_{0} / m \text { ( } m \text {-Ring Qyt. }\right)=0.6 / 3=0.2 \mathrm{~mm}
$$

Due to $A_{1}, A_{2}$ segment in the same size range, according to standard tolerances $T_{\text {avA }}$ close to IT9, according to IT9 the composition of the ring $A_{1}, A_{2}$ tolerance for $T_{1}=T_{2}=0.2 \mathrm{~mm}$, then

$$
T_{3}=T_{0}-T_{1}-T_{2}=0.6-0.2-0.2=0.2 \mathrm{~mm} \text {. }
$$

Each Composition Ring Limit Deviation. Limit deviation due to the composition of the ring into the body unknown direction, so base on the "symmetrical deviation" configuration principle, take $A_{1}=759.48 \pm 0.1 \mathrm{~mm} ; A_{2}=765.41 \pm 0.1 \mathrm{~mm} ; A_{3}=1753 \pm 0.1 \mathrm{~mm}$.

\section{Limit the Size of the Accounting Closed ring.}

$$
\begin{aligned}
& A_{0 \max }=1753.1-759.38-765.31=228.41 \mathrm{~mm} \\
& A_{0 \min }=1752.9-759.58-765.51=227.81 \mathrm{~mm}
\end{aligned}
$$

Then $A_{0}=228.11 \pm 0.3 \mathrm{~mm}$. Accounts show that the closed loop size to meet the requirements, so the $A_{1}, A_{2}, A_{3}$ take as: $A_{1}=759.48 \pm 0.1 \mathrm{~mm} ; A_{2}=765.41 \pm 0.1 \mathrm{~mm} ; A_{3}=1753 \pm 0.1 \mathrm{~mm}$.

Above value of $A_{1}, A_{2}, A_{3}$ meet to drawings, as long as their manufacturing, we can guarantee complete with mounted for qualified products.

Above Frame Assembly, for Example, Analyze the Effect of Each of $A_{1}$ Size Tolerances and Limits

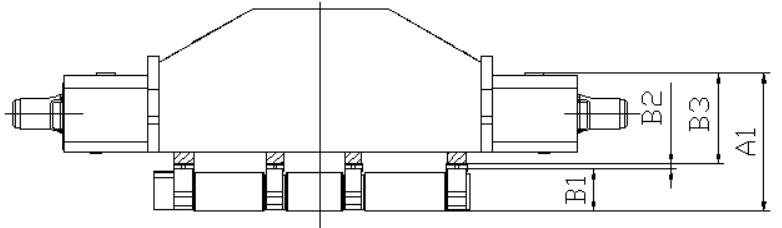

Figure.3 Inner arc frame in segement

$A_{1}$ is on the roll surface from within the framework of the arc from the plane, which determines the size of the arc-shaped roller surface precision. According to the above analysis, $A_{1}$ size requirements as $A_{1}=759.48 \pm 0.1 \mathrm{~mm}, A_{1}$ is associated with the size of $B_{1}, B_{2}, B_{3}$, shown in Figure 3 .

$B_{1}$ for the roller assembly size, which has its own size chain; $B_{2}$ for the middle plate and adjust the pad size; $B_{3}$ for the inner frame size. Analysis calculated for $A_{1}$ of three steps: Analysis calculated for $B_{1}, B_{2}, B_{3}$ of the size tolerances and limits; (2) $B_{1}$ determined after calculation and $B_{1^{-}}$related dimensional tolerances and limit deviations. (3) Comprehensive analysis of a given $A_{1}$ in each size tolerances and limits.

Analysis Calculated $\boldsymbol{B}_{1}$, Tolerances and Limits $\boldsymbol{B}_{2}, \boldsymbol{B}_{3}$. By calculation, if the components are completely interchangeable ring by assembly method selected, $B_{1}$ tolerance of $T=0.04 \mathrm{~mm}$, too strict, difficult to manufacture. So here to choose a statistical analysis to calculate interchange assembly, because in a stable process system performed when mass production and mass production, part size extremes appear unlikely. Now still ends roller Case No.1 and No.7: $B_{1}=\left(125 \times \operatorname{COS} 10^{\circ}+230 / 2\right)=238.1 \mathrm{~mm}, \quad B_{2}=\left(33 \times \operatorname{COS} 10^{\circ}\right)=32.5 \mathrm{~mm}, \quad B_{3}=488.88 \mathrm{~mm}$ (within the framework of the arc processing size), after assembly to ensure that the size of $a_{1}=759.48 \pm 0.1 \mathrm{~mm}$. Figure 4 shows the assembly dimension chain. 
$A_{1}$ is a closed loop, $B_{1}, B_{2}, B_{3}$ as plus ring, without minus ring.

The size of a closed loop is

$$
A_{1}=238.1+32.5+488.88=759.48 \mathrm{~mm}
$$

The closed loop tolerance is

$$
T_{0}=+0.1-(-0.1)=0.2 \mathrm{~mm}
$$

The average square tolerance of each component ring is

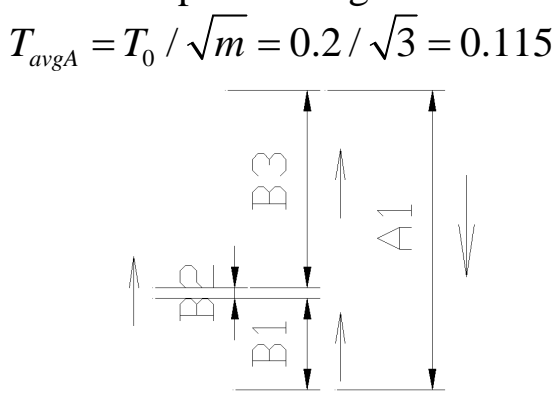

Fig.4 No.1and7 roller assembly dimension chain

Due to $B_{2}$ is small size and good processing, so define it to coordinate ring; according to $B_{1}, B_{3}$ size segment, according to the standard tolerance, $B_{1}$ by IT $9, B_{3}$ by IT8, tolerance respectively $T_{1}=0.115 \mathrm{~mm}, T_{3}=0.97 \mathrm{~mm}$, then

$$
T_{2}=\sqrt{T_{0}^{2}-T_{1}^{2}-T_{3}^{2}}=\sqrt{0.22-0.1152-0.0972}=0.132 \mathrm{~mm}
$$

Easy processing due to $B_{2}, B_{3}$ size is determined by the frame processing, frame and difficult process, so the tolerance reassigned as: $T_{1}=0.14 \mathrm{~mm}, T_{2}=0.06 \mathrm{~mm}, T_{3}=0.10 \mathrm{~mm}$.

Then

$$
T_{0}=\sqrt{T_{1}^{2}-T_{2}^{2}-T_{3}^{2}}=\sqrt{0.412-0.062-0.102}=0.182 \mathrm{~mm} .
$$

This value is less than $T_{0}=0.2 \mathrm{~mm}$.

The limit deviation of each component ring is as follows. $B_{1}$ for the roller assembly dimensions. Tentatively define $B_{1}=238.1 \pm 0.07 \mathrm{~mm} . B_{3}$ is frame sizes, finished products, one end of the size of the reference plane, and the other end of the size of the notch surface, into the direction of the body composed of the ring limit deviation is unknown, by "symmetrical bias" configuration principle, take $B_{3}=488.88 \pm 0.05 \mathrm{~mm}$. $B_{2}$ for the plate size, is the coordination ring, the final set. Due to the closed loop center deviation $\Delta_{0}=\Delta_{1}+\Delta_{2}+\Delta_{3}$, so $\Delta_{2}=\Delta_{0}-\Delta_{1}-\Delta_{3}$.

$$
\begin{gathered}
\Delta_{0}=[0.1+(-0.1)] / 2=0 \mathrm{~mm} \\
\Delta_{1}=[0.07+(-0.07)] / 2=0 \mathrm{~mm} \\
\Delta_{3}=[0.05+(-0.05)] / 2=0 \mathrm{~mm} \\
\Delta_{2}=0-0-0=0 \mathrm{~mm}
\end{gathered}
$$

Upper deviation of $B_{2}$ is

$$
E S_{2}=\Delta_{2}+T_{2} / 2=0+0.06 / 2=0.03
$$

Below deviation of $B_{2}$ is

$$
E I_{2}=\Delta_{2}-T_{2} / 2=0-0.06 / 2=-0.03
$$

Take $B_{2}=32.5 \pm 0.03 \mathrm{~mm}$, and take $B_{1}=238.1 \pm 0.07 \mathrm{~mm} ; B_{2}=32.5^{+} 0.03 \mathrm{~mm} ; B_{3}=488.88 \pm 0.05 \mathrm{~mm}$.

Accounting closed loop limit deviation is as follows.

$\Delta_{0}=\Delta_{1}+\Delta_{2}+\Delta_{3}=0+0+0=0 \mathrm{~mm}, T_{0}=\sqrt{T_{1}^{2}-T_{2}^{2}-T_{3}^{2}}=\sqrt{0.412-0.062-0.102}=0.182 \mathrm{~mm}$.

So $E S_{0}=\Delta_{0}+T_{0} / 2=0+0.182 / 2=0.091 \mathrm{~mm}, E I_{0}=\Delta_{0}-T_{0} / 2=0-0.182 / 2=-0.091 \mathrm{~mm}, A_{1}=759.48 \pm 0.091$, meet to $A_{1}=759.48 \pm 0.1 \mathrm{~mm}$.

According to accounting results, $B_{1}, B_{2}, B_{3}$ take values: $B_{1}=238.1 \pm 0.07 \mathrm{~mm} ; B_{2}=32.5 \pm 0.03 \mathrm{~mm}$; $B_{3}=488.88 \pm 0.05 \mathrm{~mm}$.

From the results, $B_{1}, B_{2}, B_{3}$ sizes, only $B_{3}$ meet to drawings, $B_{1}, B_{2}$ and the size of the drawings are not consistent, which shows, according to drawing processing and can't meet the production and quality of production requirements, according to the size of the manufacturing analysis, can guarantee to "pick up the parts on the equipment, ensure Bahrain are qualified" 
requirements, in order to achieve production goals.

Dimensional Tolerances and Limit Deviations Analysis Calculated for $B_{1}$ Influential Constituent Rings. $B_{1}=238.1$ after dimensions of the roller assembly, mounted on the portion 7 \# free rollers. Here to separate out a $\phi 250$ drive roller assembly, its size is $260 B_{1}$. The following analysis of size 260 to size instead of 238.1. Figure 5 shows the roller assembly two points, one is the lower half of the roller sleeve, bearing, spindle, bearing, size 1 at $B=135$; the second is the upper half of the mandrel and sleeve, size $B_{10 n}=250 / 2=125$. Free roller tolerance and the same drive roller, the process of several negligible analysis: the bearing only consider the inner and outer ring size tolerance, internal radial tolerances, etc.; spindle and the roller sleeve of the key parts are not accounted for only consider the mandrel diameter and tolerance of roller inner diameter.

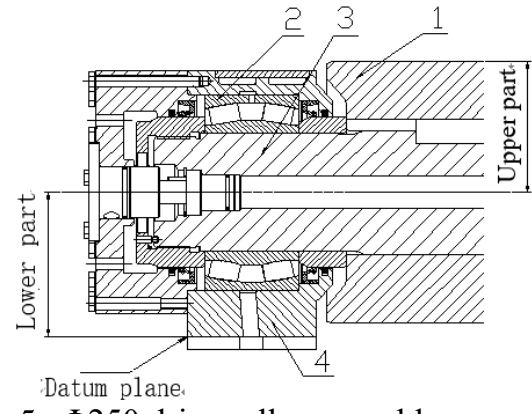

Figure.5 $\Phi 250$ drive roller assembly

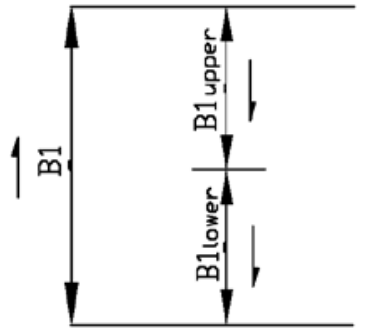

Figure.6 Assembly Dimension Chain

(1) First, $B_{1}=260 \pm 0.07 \mathrm{~mm}$ of tolerance exploded onto the lower half of the roller assembly. The allowable dimensional tolerances and limit deviations for the upper and lower roller assemblies are calculated and analyzed.

1- roller sleeve: the inner diameter of $\left.\phi=129 \mathrm{H} 7{ }_{0}^{+0.040}\right)$, the outer diameter $\phi=250_{-0.1}^{+0.1}$.

2- bearings: inner $\varphi=110_{-0.02}^{0}$, outer $\varphi=180_{-0.025}^{0}$.

3- mandrel: a large-diameter $\varphi=129 \mathrm{~h} 6\left(_{-0.025}^{0}\right)$, the small diameter $\varphi=110 \mathrm{f} 7\left(\begin{array}{c}-0.0071 \\ -0.036\end{array}\right)$.

4- bearing: bore $\varphi=180 \mathrm{H} 7\left(_{0}^{+0.04}\right)$, the base surface to the hole center distance $L=135$.

According B1 tolerance value, still choose to exchange statistical analysis to calculate the assembly, after assembly to ensure that the size of $B_{1}=260 \pm 0.07 \mathrm{~mm}$. Assembly size chain as shown in Figure 6.

$B_{1}$ is a closed loop, the $B_{1}, B_{1}$ is under increasing ring, without reducing ring.

Closed loop on the size is

The closed loop tolerance is

$$
B_{1}=B_{1}+B_{1 \text { under }}=135+250 / 2=260 \mathrm{~mm}
$$

$$
T_{0}=+0.07-(-0.07)=0.14 \mathrm{~mm}
$$

The average square tolerance of each component ring is

$$
T_{\text {avgA }}=T_{0} / \sqrt{m}=0.14 / \sqrt{2}=0.099
$$

According to the $B_{1}, B_{1}$ lower size segment, according to standard tolerances take IT9, tolerance of $T_{1}=T_{2}=0.10 \mathrm{~mm}$. Because under $\mathrm{B} 1$ chain size small, so the tolerance reassigned as: $T_{1}=0.10$ $\mathrm{mm}, T_{2}=0.09 \mathrm{~mm}$.

Then $T_{0}=\sqrt{T_{1}^{2}+T_{2}^{2}}=\sqrt{0.10^{2}+0.09^{2}}=0.135 \mathrm{~mm}$. This value is less than $T_{0}=0.14 \mathrm{~mm}$.

Constituent limit deviation ring is as following. The lower half of the roller sleeve on $B_{1}$, bearings, spindle, bearing assembly dimension, tentatively scheduled for next $B_{1}=135 \pm 0.05 \mathrm{~mm}$.

Under $B_{1}$ for the upper half of the mandrel and sleeve assembly size, tentatively scheduled for the $B_{1}=125 \pm 0.045 \mathrm{~mm}$.

Calculate the limit deviation of closed loop is

$\Delta_{0}=\Delta_{1}+\Delta_{2}=[0.05+(-0.05)] / 2+[0.045+(-0.045)] / 2=0 \mathrm{~mm}$,

$T_{0}=\sqrt{T_{1}^{2}+T_{2}^{2}}=\sqrt{0.10^{2}+0.09^{2}}=0.135 \mathrm{~mm}$,

$E S_{0}=\Delta_{0}+T_{0} / 2=0+0.135 / 2=0.07 \mathrm{~mm}$,

$E I_{0}=\Delta_{0}-T_{0} / 2=0-0.135 / 2=-0.07 \mathrm{~mm}$.It can be seen $B_{1}=260 \pm 0.07$ comply with $B_{1}=260 \pm 0.07 \mathrm{~mm}$ 
requirements. According to the accounting result, the $B_{1}, B_{1}$ lower value of: $B_{1 \text { upper }}=135 \pm 0.05 \mathrm{~mm}$; $B_{\text {1below }}=125 \pm 0.045 \mathrm{~mm}$.

(2) Under the $B_{1}=135 \pm 0.05 \mathrm{~mm}$ decomposed into the lower half of the roller assembly, $B_{1}$ lower tolerance is allowed under half of the roller assembly gap, shown in Figure 7. The lower half of the roller is equipped with two gap size, a processing size. Two gap sizes: small diameter mandrel $\varphi=110_{-0.071}^{-0.036}$ with the gap and the bearing inner ring $\varphi=110_{-0.02}^{0}$, the bearing bore $\varphi=180_{-0.025}^{0}$ and the bearing outer ring $\varphi=180_{-0.025}^{0}$ with gap; processing size have bearing center height $135 \pm 0.1$. By figure 7 calculate the size of the gap and processing resulting assembly clearance under $B_{1}$ compared with the size of the allowable gap.

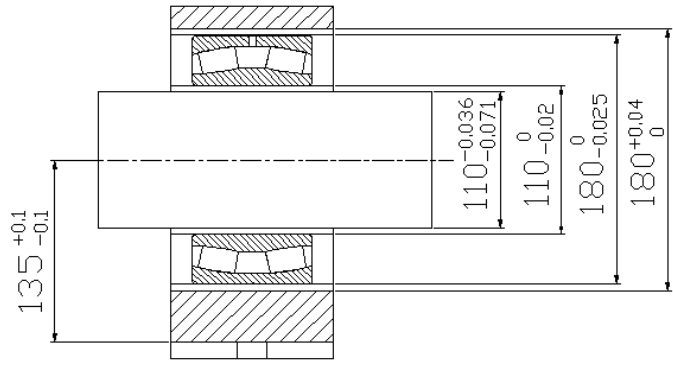

Figure.7 Lower half roller assembly clearance

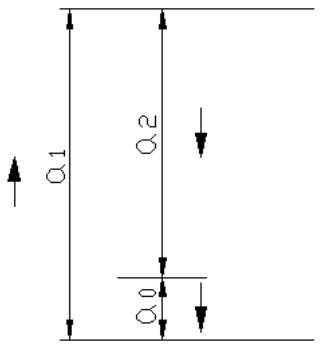

Figure.8 lower half of the roller assembly dimension chain

(1) The mandrel path $\varphi=110_{-0.071}^{-0.036}$ with the bearing inner ring $\varphi=110_{-0.02}^{0}$ tolerance fit analysis, the formation of dimensional chain Figure 8, closed loop $a_{0}=a_{1}-a_{2}, a_{\max }=0 / 2-(-0.071 / 2)=0.0355$, $a_{\min }=-0.02 / 2-(-0.036 / 2)=0.008, a_{0}=0_{+0.008}^{+0.0355}$ ( assembly clearance).

(2) Bearing inner bore $\varphi=180_{0}^{+0.04}$ and the bearing outer ring $\varphi=180_{-0.025}^{0}$ with tolerance analysis, the formation of the size of the chain as shown in Figure 9: Closed loop $a_{01}=a_{3}-a_{4}, a_{\max }=0.04 / 2-$ $(-0.025 / 2)=0.0325, a_{\min }=0 / 2-0 / 2=0, a_{01}=0_{0}^{+0.0325}$ ( assembly clearance).

(3)Bore center plane to the distance $L=135 \pm 0.1$ tolerance fit analysis. Dimension Chain see Figure 10:

Closed loop $a_{02}=a_{5}-\left(a_{0}+a_{01}\right), a_{\max }=0.1-(0.008+0)=0.092, a_{\min }=-0.1-(0.0355+0.0325)=-0.168$, $a_{02}=0_{-0.168}^{+0.092}$ (assembly tolerance value).

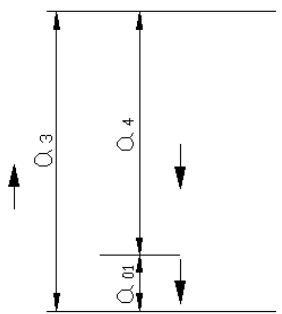

Figure.9 the bearing outer ring assembly size chain

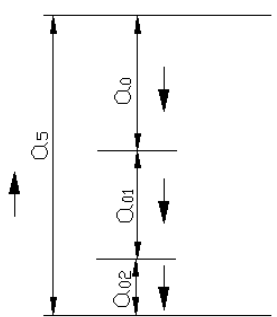

Figure.10 Base into the hole assembly dimension chain

Through the above analysis, $a_{02}=0_{-0.168}^{+0.092}$ (assembly tolerance value) $B_{1 \text { below }}=135 \pm 0.05 \mathrm{~mm}$ $\mathrm{mm}$ does not match, due to (1), (2) item change space is small, so only key to adjust (3) So $a_{02}$ and $B_{1} \quad$ under the anastomosis. By calculation, $L=135_{-0.1}^{+0.1}$ take $L=135_{+0.02}^{+0.06}$, then $a_{\max }=0.06-(0.008+0)=0.052, a_{\min }=0.02-(0.0355+0.0325)=-0.048, a_{02}=0_{-0.048}^{+0.052}$ (assembly tolerance values) $B_{1 \text { below }}=135 \pm 0.05 \mathrm{~mm}$ for meet, after adjustments ,take B1below $=135_{-0.048}^{+0.052} \mathrm{~mm}$.

(3)Decomposed $B_{1}$ upper $=125 \pm 0.045 \mathrm{~mm}$ into the upper half of the roller assembly, the $B_{1}$ upper tolerance is the upper half of the roller assembly to allow clearance shown in Figure 11. The upper half of the roller is equipped with two gap size: large diameter mandrel $\varphi=129_{-0.025}^{0}$ and the inner diameter of the roller sleeve $\varphi=129_{0}^{+0.04}$ with the gap, the spindle center to the outer diameter of the roller sleeve $\varphi=250 \pm 0.1$ with the gap. 11calculated for each by gap resulting assembly gap compared with the $B_{1}$ size allowable gap. 

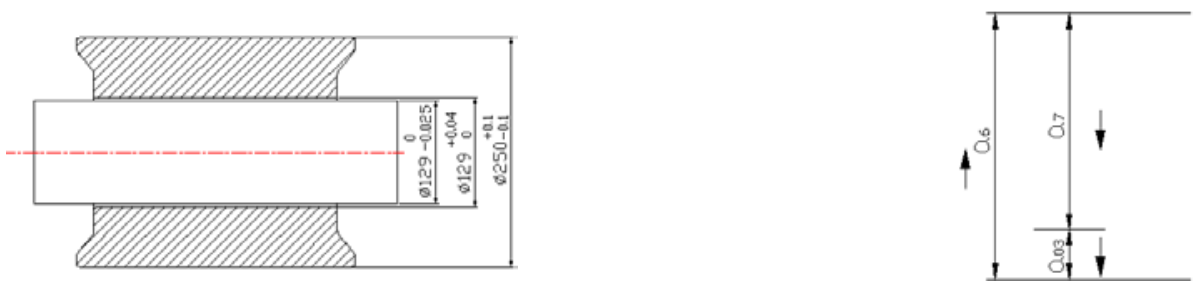

Figure.11 allow the upper half of the roller assembly gap Figure.12 large diameter mandrel assembly dimension chain

(1)For large diameter mandrel $\phi=129_{-0.025}^{0}$ and the roller sleeve inner diameter $\phi$ $=129_{0}^{+0.04}$ tolerance fit analysis, the formation of dimensional chain Figure 12: Closed loop $a_{03}=a_{6}-a_{7}, \quad a_{\max }=0.04 / 2-(-0.025 / 2)=0.0325, \quad a_{\min }=0 / 2-(0 / 2)=0, a_{03}=0+0.03250$ (assembly tolerance value).

(2) Spindle center to the outer diameter of the roller sleeve $\phi=250 \pm 0.1$ tolerance fit analysis, the formation of dimensional chain Figure 13: Closed loop $a_{04}=a_{8}-a_{03}, \quad a_{\max }=0.1 / 2-0=0.05$, $a_{\min }=-0.1 / 2-(0.0325)=-0.0825, \quad a_{04}=0_{-0.0825}^{+0.05} \quad$ (assembly clearance). Through the above analysis, $a_{04}=0_{-0.0825}^{+0.05}$ (Assembly tolerance value) And on $B_{1}=125 \pm 0.045 \mathrm{~mm}$ do not coincide, because (1) Term changes very little space, so only key to make the adjustment (2) $a_{04}$ and $B_{1}$ match. By calculation, the outer diameter of the roller sleeve by the $\phi=250 \pm 0.1$ take $\phi$ $=250_{-0.025}^{+0.09}$ then $a_{\max }=0.09 / 2-0=0.045, a_{\min }=-0.025 / 2-(0.0325)=-0.045, a_{04}=0_{-0.045}^{+0.045}$ ( assembly tolerances) and $B_{1}$. on $=125 \pm 0.045 \mathrm{~mm}$ fit, take the $B_{1}$ at cost: the $B_{1}=125 \pm 0.045 \mathrm{~m}$.

(4)Take the set $B_{1}=135_{-0.048}^{+0.052} \mathrm{~mm}, B_{1}=125 \pm 0.045 \mathrm{~mm}$, closed loop $B_{1}$ accounting limit deviation is

$$
\begin{gathered}
\Delta_{0}=\Delta_{1}+\Delta_{2}=[0.052+(-0.048)] / 2+[0.045+(-0.045)] / 2=0.002 \mathrm{~mm}, \\
T_{0}=\sqrt{T_{1}^{2}+T_{2}^{2}}=\sqrt{0.10^{2}+0.09^{2}}=0.135 \mathrm{~mm},
\end{gathered}
$$

So

$$
\begin{gathered}
E S_{0}=\Delta_{0}+T_{0} / 2=0.002+0.135 / 2=0.07 \mathrm{~mm} \\
E I_{0}=\Delta_{0}-T_{0} / 2=0.002-0.135 / 2=-0.07 \mathrm{~mm}
\end{gathered}
$$

Seen $B_{1}=260 \pm 0.07$ in line with $B_{1}=260 \pm 0.07 \mathrm{~mm}$ requirements.

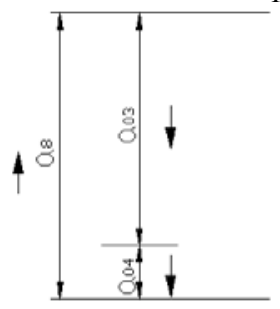

Figure .13 Roller sleeve assembly dimension chain

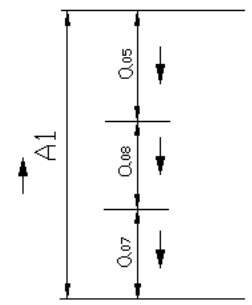

Fig.14 on the frame assembly tolerances

Upper frame assembly tolerance analysis. The frame assembly tolerance A1 main factors are B1 namely $a_{05}, B_{2}$ namely $a_{08}$ (intermediate plate tolerance of \pm 0.05 ), $B_{3}$ namely $a_{07}$ (bearing surface to the frame plane tolerance of $\pm 0.05 \mathrm{~mm}$ ).

$$
\begin{gathered}
A_{1}=a_{05}+a_{08}+a_{07}, A_{1 \max }=0.142+0.05+0.05=0.242 \\
A_{1 \min }=-0.2505-0.05-0.05=-0.1505, A_{1}=0_{-0.2505}^{+0.242}
\end{gathered}
$$

Thus, the tolerance value is also greater than the upper frame drawings of $\pm 0.15 \mathrm{~mm}$.

\section{Segment assembly quality assurance process measures}

In order to ensure the precision of the assembly process will be given to drawing tolerances sharing, mainly to be adjusted for large impact factors. Adjustment principle is: the tolerance for the large coefficient of freedom to be open, simple and easy to deal with the tolerance given to the provisions. From the above tolerance analysis, the main impact factor is the roller assembly $B_{1}$ (roller assembly tolerance $a_{05}$ ), while the main factors affecting $B_{1}, a_{02}, a_{04}$. The following is a list of the tolerances given to the specified parts. 
Roller Assembly Control Measures. (1) Roller assembly tolerances $\left(a_{05}\right)$ controlled by $a_{05}=0_{-0.2505}^{+0.142}$ becomes $a_{05}=0_{-0.0805}^{+0.072}$. Roller sleeve diameter tolerance of $\varphi \pm 0.1$ is defined as $\varphi$ ${ }_{0}^{+0.06}$.Bearing center hole to the bottom plane tolerance $L \pm 0.1$ is defined as $\mathrm{L}_{+0.02}^{+0.05}$. Change dimension chain Figure 15:

(1) Base into the hole center distance $L$ size chain $a_{02}$ : closed loop $a_{02}=a_{5}-\left(a_{0}+a_{01}\right)$, $a_{\max }=0.05-(0.008+0)=0.042, a_{\min }=0.02-(0.0355+0.0325)=-0.048, a_{02}=0_{-0.048}^{+0.042}$.

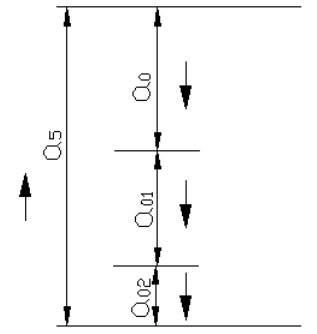

Fig.15 bearing center whole assembly dimension chain

(2) Spindle center to the outer diameter of the roller sleeve chain a04: closed loop $a_{04}=a_{8}-a_{03}$, $a_{\max }=0.06 / 2-0=0.03, a_{\min }=0 / 2-(0.0325)=-0.0325, a_{04}=0+0.03-0.0325$.

(3) Roller assembly tolerance closed loop is: $a_{05}=a_{02}+a_{04}, \quad a_{\max }=0.042+0.03=0.072$, $a_{\text {min }}=-0.048+(-0.0325)=-0.0805, a_{05}=0_{-0.0805}^{+0.072}$.

(2)On the frame tolerance $B_{3}\left(a_{07}\right)$ controlled $a_{07_{-0}^{+0.03}}$; (3) intermediate plate tolerance $B_{2}\left(a_{08}\right)$ controlled $a_{08-0.01}^{+0.01}$; (4) tolerance value adjusted $A_{1}$.

$$
\begin{gathered}
A_{1}=a_{05}+a_{08}+a_{07} \\
A_{1 \max }=0.072+0.01+0.03=0.112 \\
A_{1 \text { min }}=-0.0805-0.01-0=-0.0905 \\
A_{1}=0_{-0.0905}^{+0.112} \text { meet the requirements of the drawings } \pm 0.15 \mathrm{~mm}
\end{gathered}
$$

Process Control Points. (1) Bearing the center hole to the bottom plane tolerance $L \pm 0.1$ is defined $L_{+0.02}^{+0.05}$. (2) Roller sleeve diameter tolerance $\Phi \pm 0.1$ is defined as $\Phi_{0}^{+0.06}$. (3) On the frame tolerance $Y \pm 0.05$ That $B_{3}\left(a_{07}\right)$ controlled $a_{07-0}^{+0.03}$. (4) An intermediate plate Tolerance $T \pm 0.05$ is $B_{2}$ (a08) controlled $a_{08} \pm 0.01$.

\section{Conclusion}

After the above process analysis and calculations, assembly process billets key segments of the size of the opening height the guarantee, at the same time by calculating that each roller assembly quality has been effectively guaranteed, process control measures are in line with the quality requirements for the sector Section of the correct assembly laid a foundation.

\section{References}

[1]Zhen Liyan. Segment manufacturing process quality control. A heavy technology, 2007.11

[2]Beijing Institute of Mechanical Engineering of Manufacturing Engineering, Beijing: China's Institute of Mechanical Engineering Technology Management Committee publish, 2001.1

[3] Beijing Mechanical Engineering Society. Modern manufacturing engineering. Beijing: China Machinery Engineering Society Process Management Professional Committee.2001.1

[4] "Mechanical Design Manual" writing group. Machine Design Manual Volume III. China Machine Press,1986.12

[5] Wu Zongze. Mechanical Design Manual I. Beijing: China Machine Press,2002

[6] Wu Zongze. Mechanical Design Manual 2. Beijing: China Machine Press,2002

[7] China Mechanical Engineering Society. China Mechanical Design Canon. Jiangxi :Jiangxi 
science and Technology Press,2002 